\section{STROMAL CELL SIALYLATION SUPPRESSES T CELLS IN INFLAMMATORY TUMOUR MICROENVIRONMENTS: A NEW TUMOUR STROMAL CELL IMMUNE CHECKPOINT?}

${ }^{1}$ Oliver Treacy*, 'Hannah Egan, ${ }^{1}$ Kevin Lynch, ${ }^{1}$ Niamh Leonard, ${ }^{2}$ Kim De Veirman, ${ }^{2}$ Karin Vanderkerken, ${ }^{1}$ Laurence Egan, ${ }^{1}$ Thomas Ritter, ${ }^{3}$ Aisling Hogan, ${ }^{4}$ Keara Redmond, ${ }^{1} J a n u s z$ Krawczyk, ${ }^{1}$ Sean Hynes, ${ }^{4}$ Emma Kerr, ${ }^{4}$ Philip Dunne, ${ }^{1}$ Michael O'Dwyer, ${ }^{1}$ Aideen Ryan. 'National University of Ireland, Galway, Galway, Ireland; ' ${ }^{2}$ Vrije Universiteit Brussel, Brussels, Belgium; ${ }^{3}$ Galway University Hospital, Galway, Ireland; ${ }^{4} Q u e e n ' s$ University Belfast, Belfast, UK

Background Immunosuppressive tumour microenvironments (TME) reduce the effectiveness of immune responses in cancer. Non-haematopoietic mesenchymal stromal cells, precursors to cancer-associated fibroblasts (CAFs), dictate tumour progression by enhancing immune cell suppression. Sialic acids, which exist as terminal sugars of glycans (known as sialoglycans), are highly expressed on cancer cells and hyper-sialylation of glycans is known to promote immune evasion in cancer. Sialoglycans are recognized by sialic acid-binding immunoglobulin-like lectins (Siglecs), a family of immunomodulatory receptors, which are analogous to the immune checkpoint inhibitor PD1. ${ }^{1}$ The role of sialyation in stromal cell-mediated immunosuppression, however, is unknown. Using models of solid (colorectal cancer - CRC) and haematological (multiple myeloma MM) stromal-rich tumours in both mouse and human, the aim of this study was to investigate if stromal cell sialylation contributes to enhanced immunosuppression in the TME.

Methods Flow cytometric analysis of sialic acid expression was performed initially on bone marrow-derived stromal cells isolated from healthy human donor bone marrow aspirates, from wild-type Balb/c mice or from 5T33 multiple myeloma mice. Stromal cells were also isolated and expanded from colorectal cancer patient tumour biopsies (CAFs) with matched controls isolated from tumour-adjacent non-cancerous tissue (normalassociated fibroblasts - NAFs) or from whole blood from primary multiple myeloma bone aspirates. Informed consent was obtained from all patients prior to sampling. Immunosuppression assays were performed using these stromal cells with or without exposure to the tumour cell secretome from the mouse and human CRC cell lines CT26 or HCT116 and HT29, respectively, co-cultured with either murine lymphocytes or healthy human donor-derived peripheral blood mononuclear cells (PBMCs).

Results Our results showed that tumour conditioned stromal cells have increased levels of sialyltransferase gene expression, $\alpha 2,3 / \alpha 2,6$-linked sialic acid and Siglec ligands. Co-culture assays revealed that CAFs induced significantly higher frequencies of Siglec 7 and Siglec 9-expressing CD8 T cells, as well as Tim-3 and PD-1-expressing CD8 T cells, compared to NAFs. Inhibition of sialyltransferase activity using the inhibitor 3FAXNeu5Ac reversed these CAF-induced effects. Interestingly, sialyltransferase inhibition had no observed effects on $\mathrm{T}$ cells co-cultured with NAFs.

Conclusions These results demonstrate that targeting stromal cell sialylation can reverse immune cell suppression and reactivate exhausted $\mathrm{T}$ cells. These novel data support a rationale for the assessment of stromal cell sialylation and Siglec ligand expression in order to better stratify patients for immunotherapeutic combination treatments that aim to reactivate exhausted $\mathrm{T}$ cells in stromal-enriched tumour microenvironments.

Acknowledgements The authors would like to thank the Blood Cancer Network of Ireland Biobank for providing bone marrow aspirates.

\section{REFERENCE}

1. Gray MA, Stanczak MA, Mantuano NR, Xiao H, Pijnenborg JFA, Malaker SA, Miller CL, Weidenbacher PA, Tanzo JT, Ahn G, Woods EC, Läubli H, Bertozzi CR Targeted glycan degradation potentiates the anticancer immune response in vivo. Nat Chem Biol 2020;16:1376-1384.

Ethics Approval Colorectal tumor and adjacent normal mucosal tissue were obtained from patients undergoing colon tumor resection at University Hospital Galway under an ethically approved protocol (Clinical Research Ethics Committee, Ref: C.A. 2074). Samples were collected and isolated by the Blood Cancer Network of Ireland under an ethically approved protocol. Written informed explicit consent was obtained from all patients prior to sampling. Mice were housed and maintained following the conditions approved by the Animals Care Research Ethics Committee of the National University of Ireland, Galway (NUIG) and procedures were conducted under individual and project authorisation licenses from the Health Products Regulatory Authority (HPRA) of Ireland or from the Ethical Committee for Animal Experiments, Vrije Universiteit Brussel (license no. LA1230281, 16-281-6).

http://dx.doi.org/10.1136/jitc-2021-SITC2021.941 